\title{
Synthesis and Biological Evaluation of Novel Piperazine Containing Hydrazone Derivatives
}

\author{
Betül Kaya, ${ }^{1}$ Yusuf Özkay, ${ }^{1,2}$ Halide Edip Temel, ${ }^{3}$ and Zafer Asım Kaplancıklı1 \\ ${ }^{1}$ Faculty of Pharmacy, Department of Pharmaceutical Chemistry, Anadolu University, 26470 Eskişehir, Turkey \\ ${ }^{2}$ Faculty of Pharmacy, Doping and Narcotic Compounds Analysis Laboratory, Anadolu University, 26470 Eskişehir, Turkey \\ ${ }^{3}$ Faculty of Pharmacy, Department of Biochemistry, Anadolu University, 26470 Eskişehir, Turkey \\ Correspondence should be addressed to Yusuf Özkay; yozkay@anadolu.edu.tr
}

Received 1 March 2016; Revised 22 June 2016; Accepted 17 July 2016

Academic Editor: Patricia Valentao

Copyright (C) 2016 Betül Kaya et al. This is an open access article distributed under the Creative Commons Attribution License, which permits unrestricted use, distribution, and reproduction in any medium, provided the original work is properly cited.

\begin{abstract}
Some hydrazone derivatives were synthesized and their potential anticholinesterase activities were examined. A series of eleven new compounds of $\mathrm{N}^{\prime}$-(2,4-disubstitutedbenzylidene)-2-(4-(4-nitrophenyl)piperazin-1-yl)acetohydrazide derivatives were obtained via reaction of 2-[4-(4-nitrophenyl)piperazin-1-yl] acetohydrazide with aromatic aldehydes. The chemical structures of the compounds were enlightened by FT-IR, ${ }^{1} \mathrm{H}-\mathrm{NMR},{ }^{13} \mathrm{C}-\mathrm{NMR}$, and HRMS (ESI) spectral data. The inhibition potency of the compounds $\mathbf{3 a}-\mathbf{k}$ against $\mathrm{AChE}$ and $\mathrm{BuChE}$ was measured and evaluated using a modification of Ellman's spectrophotometric method. Among the tested compounds, compound $3 \mathrm{c}$ was assigned to be the most active derivative. Galantamine was used as a standard drug.
\end{abstract}

\section{Introduction}

Alzheimer's disease $(\mathrm{AD})$ is a chronic neurodegenerative and progressive disease responsible from $60 \%$ to $70 \%$ of cases of dementia [1] and characterized by progressive impairment of memory and cognition [2]. The primary lesions of $\mathrm{AD}$ are neurofibrillary tangles (NFT) and the senile plaques that is composed of central core of beta-amyloid [3,4]. As the destruction of cholinergic neurons ends up with the decline in acetylcholine (ACh) level, a key aspect of the symptomatic therapy for $\mathrm{AD}$ is to increase acetylcholine concentration in presynaptic regions via blocking its metabolic enzyme acetylcholinesterase (AChE). In humans, cholinesterase is classified into two types: acetylcholinesterase (AChE) that hydrolyses acetylcholine selectively and more quickly and butyrylcholinesterase $(\mathrm{BuChE})$ that metabolize butyrylcholine more quickly $[5,6]$. The therapeutic approaches to date have tended to focus on $\mathrm{AChE}$ rather than BuChE that is considered to play a minor role in regulating ACh levels in brain. At the present time, reversible acetylcholinesterase inhibitors (AChEIs) like donepezil, rivastigmine, and galantamine are in clinical use in the therapy of AD as well as the N-methylD-aspartate-receptor (NMDA) antagonist memantine $[7,8]$.
Reversible AChE inhibitors perform a significant role in pharmacological manipulation of the enzyme activity [9]. Despite their efficacy, reversible AChE inhibitors suffer from several major drawbacks such as hepatotoxicity, short half life, and gastrointestinal tract excitement [10]. For medicinal chemists, hence, it has been an increasingly important area to discover novel and efficient AChE inhibitors with minimum side effects.

The past decades have seen increasingly rapid advances in the field of developing acetylcholinesterase inhibitors. Hydrazones, thus, have attracted great attention in the light of recent studies that reported compounds bearing hydrazone moiety exhibit various biological activities including acetylcholinesterase activity [11-18]. On the other hand, piperazine ring is known to act as bioisosteric replacement for piperidine ring that exist in donepezil and have also been revealed to have acetylcholinesterase activity [19-21]. Based on these observations, we designed a new series of piperazine containing hydrazone derivatives (Figure 1). This combination of studies provides support for designing novel and efficient agents for treatment of AD.

In view of the above-mentioned findings, herein, we reported the synthesis of and acetylcholinesterase 
and butyrylcholinesterase inhibitory activity of $\mathrm{N}^{\prime}-(2,4-$ disubstitutedbenzylidene)-2-(4-(4-nitrophenyl)piperazin-1yl)acetohydrazide derivatives that may be of value in designing acetylcholinesterase inhibitors. The inhibitory potency of compounds was studied in comparison with the known AChE inhibitor drug galantamine.

\section{Experimental}

2.1. Materials. All chemicals were purchased from SigmaAldrich Chemical Co. (Sigma-Aldrich Corp., St. Louis, MO, USA) and Merck Chemicals (Merck KGaA, Darmstadt, Germany). All melting points ( $\mathrm{mp}$ ) were determined by Electrothermal 9100 digital melting point apparatus (Electrothermal, Essex, UK) and were uncorrected. All the reactions were monitored by thin-layer chromatography (TLC) using Silica Gel 60 F254 TLC plates (Merck KGaA, Darmstadt, Germany). Spectroscopic data were recorded with the following instruments: IR, Shimadzu 8400S spectrophotometer (Shimadzu, Tokyo, Japan); NMR, Bruker DPX 500 NMR spectrometer (Bruker Bioscience, Billerica, MA, USA), in DMSO- $d_{6}$, using TMS as internal standard; $M+1$ peaks were determined by LC-MS-IT-TOF system (Shimadzu, Tokyo, Japan).

2.2. General Procedure for Ethyl 2-[4-(4-nitrophenyl)piperazin-1-yl]acetate (1). 1-(4-Nitrophenyl)piperazine (0.015 mol) was dissolved in acetone $(250 \mathrm{~mL})$. Potassium carbonate $(0.018 \mathrm{~mol})$ was added. After addition of ethyl 2 -chloroacetate $(0.015 \mathrm{~mol})$, the reaction mixture was stirred for 4 hours at room temperature. The solvent was evaporated under reduced pressure and then water was added to wash the resulting solid and the mixture was filtered to give compound 1.

2.3. General Procedure for 2-[4-(4-Nitrophenyl)piperazin-1yl]acetohydrazide (2). Ethyl 2-[4-(4-nitrophenyl)piperazin$1-y l]$ acetate $(0.013 \mathrm{~mol})$ was dissolved in ethanol $(250 \mathrm{~mL})$. Hydrazine hydrate $(0.013 \mathrm{~mol})$ dissolved in ethanol was added gradually and the mixture was stirred at room temperature. After completion of the reaction, the solvent was evaporated under reduced pressure; then water was added to wash the resulting solid and the mixture was filtered, dried, and recrystallized from ethanol to give compound 2.

2.4. General Procedure for $N^{\prime}$-(2,4-Disubstitutedbenzylidene)2-(4-(4-nitrophenyl)piperazin-1-yl)acetohydrazide Derivatives $(\mathbf{a} \boldsymbol{a}-\boldsymbol{k})$. 2-[4-(4-Nitrophenyl)piperazin-1-yl]acetohydrazide $(0.7 \mathrm{mmol})$ was dissolved in ethanol $(100 \mathrm{~mL})$. After addition of acetic acid $(1 \mathrm{~mL})$ and appropriate aldehydes $(0.7 \mathrm{mmol})$, the reaction mixture was refluxed for 8 hours. After TLC screening, at the end of this period, the reaction mixture was cooled, filtered, and recrystallized from ethanol to give the target compounds $\mathbf{3 a}-\mathbf{k}$.

2.4.1. $N^{\prime}$-(4-Fluorobenzylidene)-2-(4-(4-nitrophenyl)piperazin-1-yl)acetohydrazide (3a). IR (KBr) $v_{\max }\left(\mathrm{cm}^{-1}\right): 3259$ (N-H), 3066 (Aromatic C-H), 2885 (Aliphatic C-H), 1695
$(\mathrm{C}=\mathrm{O}), 1597(\mathrm{C}=\mathrm{N}), 1581$ and $1305\left(\mathrm{NO}_{2}\right), 1473(\mathrm{C}=\mathrm{C}), 1230$ (C-N), 1143, 1076 and 827 (C-H out of plane deformation).

${ }^{1} \mathrm{H}-\mathrm{NMR}$ (400 Mhz, DMSO- $\left.d_{6}, \mathrm{ppm}\right) \delta 2.61(2 \mathrm{H}, \mathrm{t}, J=$ $4.4 \mathrm{~Hz}$, piperazine protons), $2.70(2 \mathrm{H}, \mathrm{t}, J=4.4 \mathrm{~Hz}$, piperazine protons), 3.15 and $3.61\left(2 \mathrm{H}\right.$, two s, $\left.\mathrm{CO}-\mathrm{CH}_{2}\right), 3.44-3.49(4 \mathrm{H}$, $\mathrm{m}$, piperazine protons), $6.97\left(2 \mathrm{H}, \mathrm{dd}, J_{1}=9.2 \mathrm{~Hz}, J_{2}=4 \mathrm{~Hz}\right.$, phenyl protons), 7.20-7.27 (2H, $\mathrm{m}$, phenyl photons), 7.66-7.73 ( $2 \mathrm{H}, \mathrm{m}$, phenyl protons), 7.93 and 8.31 (1H, two s, $\mathrm{N}=\mathrm{CH}), 8.01$ $\left(2 \mathrm{H}, \mathrm{dd}, J_{1}=9.2 \mathrm{~Hz}, J_{2}=3.6 \mathrm{~Hz}\right.$, phenyl protons $), 11.23$ and $11.32(1 \mathrm{H}$, two s, NH).

${ }^{13} \mathrm{C}-\mathrm{NMR}$ (100 Mhz, DMSO- $d_{6}$, ppm) $\delta 46.17$ and 46.37 $\left(2 \mathrm{CH}_{2}\right), 52.03$ and $52.19\left(2 \mathrm{CH}_{2}\right), 56.85$ and $60.07\left(\mathrm{COCH}_{2}\right)$, 112.47 and $112.55(2 \mathrm{CH}), 115.68$ and $115.90(2 \mathrm{CH}), \overline{12} 5.63$ $(2 \mathrm{CH}), 128.75$ and $128.83(\mathrm{CH}), 129.07$ and $129.16(\mathrm{CH}), 130.71$, 130.73 and $130.80,130.83(\mathrm{C}), 136.72$ and $136.83(\mathrm{C}), 141.69$ and $146.04(\mathrm{C}=\mathrm{N}), 154.64(\mathrm{C}), 161.60,161.79$ and $164.07,164.25(\mathrm{C}-$ $\mathrm{F}), 165.45$ and $170.63(\mathrm{C}=\mathrm{O})$.

HRMS $(m / z):[\mathrm{M}+\mathrm{H}]^{+}$calcd for $\mathrm{C}_{19} \mathrm{H}_{20} \mathrm{FN}_{5} \mathrm{O}_{3}$ : 386.1623; found 386.1618 .

2.4.2. $N^{\prime}$-(4-Methoxybenzylidene)-2-(4-(4-nitrophenyl)piperazin-1-yl)acetohydrazide (3b). IR (KBr) $v_{\max }\left(\mathrm{cm}^{-1}\right): 3271$ (N-H), 3070 (Aromatic C-H), 2835 (Aliphatic C-H), 1681 $(\mathrm{C}=\mathrm{O}), 1581(\mathrm{C}=\mathrm{N}), 1504$ and $1303\left(\mathrm{NO}_{2}\right), 1471(\mathrm{C}=\mathrm{C}), 1240$ (C-N), 1207-1078 (C-O), 1078 and 825 (C-H out of plane deformation).

${ }^{1} \mathrm{H}-\mathrm{NMR}$ (400 Mhz, DMSO- $\left.d_{6}, \mathrm{ppm}\right) \delta 2.64(2 \mathrm{H}, \mathrm{t}, J=$ $4.8 \mathrm{~Hz}$, piperazine protons $), 2.73(2 \mathrm{H}, \mathrm{t}, J=4.8 \mathrm{~Hz}$, piperazine protons), 3.17 and $3.64\left(2 \mathrm{H}, 2 \mathrm{~s}, \mathrm{CO}-\mathrm{CH}_{2}\right), 3.48(2 \mathrm{H}, \mathrm{t}, J=$ $4.8 \mathrm{~Hz}$, piperazine protons), $3.52(2 \mathrm{H}, \mathrm{t}, J=4.8 \mathrm{~Hz}$, piperazine protons), $3.80\left(3 \mathrm{H}, \mathrm{d}, J=3.6 \mathrm{~Hz}, \mathrm{OCH}_{3}\right), 6.98-7.04(4 \mathrm{H}, \mathrm{m}$, phenyl protons), 7.59-7.65 (2H, m, phenyl protons), 7.92 and $8.28(1 \mathrm{H}$, two s, $\mathrm{N}=\mathrm{CH}), 8.05\left(2 \mathrm{H}, \mathrm{dd}, J_{1}=9.2 \mathrm{~Hz}, J_{2}=3.6 \mathrm{~Hz}\right.$, phenyl protons), 11.12 and $11.22(1 \mathrm{H}$, two s, NH).

${ }^{13}$ C-NMR (100 Mhz, DMSO- $d_{6}$, ppm) $\delta 46.17$ and 46.36 $\left(2 \mathrm{CH}_{2}\right), 52.03$ and $52.18\left(2 \mathrm{CH}_{2}\right), 55.19\left(\mathrm{OCH}_{3}\right), 56.86$ and $60.08\left(\mathrm{COCH}_{2}\right), 112.45$ and $112.54(2 \mathrm{CH}), 114.22(2 \mathrm{CH})$, $125.63(2 \mathrm{CH}), 126.70$ and $126.73(\mathrm{C}), 128.20$ and $128.55(2 \mathrm{CH})$, 136.69 and $136.81(\mathrm{C}), 142.71$ and $147.04(\mathrm{C}=\mathrm{N}), 154.60$ and $154.65(\mathrm{C}), 160.49$ and $160.71(\mathrm{C}), 165.13$ and $170.37(\mathrm{C}=\mathrm{O})$.

$\operatorname{HRMS}(m / z):[\mathrm{M}+\mathrm{H}]^{+}$calcd for $\mathrm{C}_{20} \mathrm{H}_{23} \mathrm{~N}_{5} \mathrm{O}_{4}: 398.1823$; found 398.1838 .

2.4.3. $N^{\prime}$-(4-Hydroxybenzylidene)-2-(4-(4-nitrophenyl)piperazin-1-yl)acetohydrazide (3c). IR (KBr) $v_{\max }\left(\mathrm{cm}^{-1}\right): 3250$ (N-H), 3115.04 (Aromatic C-H), 2823 (Aliphatic C-H), 1651 $(\mathrm{C}=\mathrm{O}), 1595$ and $1305\left(\mathrm{NO}_{2}\right), 1504(\mathrm{C}=\mathrm{N}), 1473(\mathrm{C}=\mathrm{C}), 1238$ (C-N), 1116 and 823 (C-H out of plane deformation).

${ }^{1} \mathrm{H}-\mathrm{NMR}$ (400 Mhz, DMSO- $\left.d_{6}, \mathrm{ppm}\right) \delta 2.64(2 \mathrm{H}, \mathrm{t}, J$ $=4.4 \mathrm{~Hz}$, piperazine protons $), 2.71-2.76(2 \mathrm{H}, \mathrm{m}$, piperazine protons), 3.15 and $3.62\left(2 \mathrm{H}\right.$, two s, $\left.\mathrm{CO}-\mathrm{CH}_{2}\right), 3.48-3.52(4 \mathrm{H}$, $\mathrm{m}$, piperazine protons), $6.80-6.85(2 \mathrm{H}, \mathrm{m}$, phenyl protons), $7.00-7.05$ (2H, m, phenyl protons), 7.35-7.60 (2H, m, phenyl protons), 7.84 and $8.22(1 \mathrm{H}$, two s, $\mathrm{N}=\mathrm{CH}), 8.05\left(2 \mathrm{H}, \mathrm{dd}, J_{1}=\right.$ $9.6 \mathrm{~Hz}, J_{2}=2.4 \mathrm{~Hz}$, phenyl-H), $9.85(1 \mathrm{H}$, br s, OH), 11.04 and $11.14(1 \mathrm{H}$, two s, NH).

${ }^{13} \mathrm{C}-\mathrm{NMR}$ (100 Mhz, DMSO- $\left.d_{6}, \mathrm{ppm}\right) \delta 46.16$ and 46.35 $\left(2 \mathrm{CH}_{2}\right), 52.03$ and $52.16\left(2 \mathrm{CH}_{2}\right), 56.85$ and $60.07\left(\mathrm{COC}_{2}\right)$, 
112.44 and $112.53(2 \mathrm{CH}), 115.59(2 \mathrm{CH}), 125.10$ and $125.13(\mathrm{C})$, $125.63(2 \mathrm{CH}), 128.33$ and $128.70(2 \mathrm{CH}), 136.68$ and $136.79(\mathrm{C})$, 143.15 and $147.45(\mathrm{C}=\mathrm{N}), 154.60$ and $154.65(\mathrm{C}), 159.05$ and $159.29(\mathrm{C}), 165.00$ and $170.24(\mathrm{C}=\mathrm{O})$.

$\operatorname{HRMS}(m / z):[\mathrm{M}+\mathrm{H}]^{+}$calcd for $\mathrm{C}_{19} \mathrm{H}_{21} \mathrm{~N}_{5} \mathrm{O}_{4}: 384.1666$; found 384.1650 .

2.4.4. $N^{\prime}$-(4-Ethoxybenzylidene)-2-(4-(4-nitrophenyl)piperazin-1-yl)acetohydrazide (3d). IR (KBr) $v_{\max }\left(\mathrm{cm}^{-1}\right): 3253$ (N-H), 3069 (Aromatic C-H), 2976 (Aliphatic C-H), 1693 $(\mathrm{C}=\mathrm{O}), 1598.99(\mathrm{C}=\mathrm{N}), 1585$ and $1305\left(\mathrm{NO}_{2}\right), 1477(\mathrm{C}=\mathrm{C})$, 1240 (C-N), 1207-1041 (C-O), 829 (C-H out of plane deformation).

${ }^{1} \mathrm{H}-\mathrm{NMR}\left(400 \mathrm{Mhz}, \mathrm{DMSO}-d_{6}, \mathrm{ppm}\right) \delta 1.31(3 \mathrm{H}, \mathrm{t}, J=$ $\left.7 \mathrm{~Hz}, \mathrm{CH}_{3}\right), 2.61(2 \mathrm{H}, \mathrm{t}, J=4.4 \mathrm{~Hz}$, piperazine protons), 2.70 $(2 \mathrm{H}, \mathrm{t}, J=4.8 \mathrm{~Hz}$, piperazine protons $), 3.12$ and $3.59(2 \mathrm{H}$, $\left.2 \mathrm{~s}, \mathrm{CO}-\mathrm{CH}_{2}\right), 3.27(1 \mathrm{H}, \mathrm{s}$, piperazine proton), $3.42-3.60(3 \mathrm{H}$, $\mathrm{m}$, piperazine protons), $4.00-4.06\left(2 \mathrm{H}, \mathrm{m}, \mathrm{OCH}_{2}\right), 6.92-7.04$ ( $4 \mathrm{H}, \mathrm{m}$, phenyl protons), $7.54-7.62$ ( $2 \mathrm{H}, \mathrm{m}$, phenyl protons), 7.88 and $8.24(1 \mathrm{H}$, two s, $\mathrm{N}=\mathrm{CH}), 8.03\left(2 \mathrm{H}\right.$, dd, $J_{1}=9.6 \mathrm{~Hz}, J_{2}$ $=3.2 \mathrm{~Hz}$, phenyl protons), 11.03 and $11.13(1 \mathrm{H}$, two s, $\mathrm{NH})$.

${ }^{13}$ C-NMR (100 Mhz, DMSO- $\left.d_{6}, \mathrm{ppm}\right) \delta 14.47\left(\mathrm{CH}_{3}\right)$, 46.21 and $46.40\left(2 \mathrm{CH}_{2}\right), 52.02$ and $52.17\left(2 \mathrm{CH}_{2}\right), 56.84$ and $60.07\left(\mathrm{COCH}_{2}\right), 63.17\left(\mathrm{OCH}_{2}\right), 112.49$ and $112.57(2 \mathrm{CH})$, $114.65(2 \mathrm{CH}), 125.60(2 \mathrm{CH}), 126.61(\mathrm{C}), 128.18$ and 128.52 $(2 \mathrm{CH}), 136.74$ and $136.85(\mathrm{C}), 142.73$ and $147.08(\mathrm{C}=\mathrm{N}), 154.62$ (C), 159.79 and $160.01(\mathrm{C}), 165.08$ and $170.33(\mathrm{C}=\mathrm{O})$.

HRMS $(m / z):[\mathrm{M}+\mathrm{H}]^{+}$calcd for $\mathrm{C}_{21} \mathrm{H}_{25} \mathrm{~N}_{5} \mathrm{O}_{4}$ : 412.1979; found 412.1971 .

2.4.5. $N^{\prime}$-(2,4-Dichlorobenzylidene)-2-(4-(4-nitrophenyl)piperazin-1-yl)acetohydrazide (3e). IR (KBr) $\nu_{\max }\left(\mathrm{cm}^{-1}\right): 3259(\mathrm{~N}-$ $\mathrm{H}), 3093$ (Aromatic C-H), 2862 (Aliphatic C-H), 1683 (C=O), 1597 and $1303\left(\mathrm{NO}_{2}\right), 1508(\mathrm{C}=\mathrm{N}), 1490(\mathrm{C}=\mathrm{C}), 1232(\mathrm{C}-\mathrm{N})$, 1099 and 821 (C-H out of plane deformation).

${ }^{1} \mathrm{H}-\mathrm{NMR}\left(400 \mathrm{Mhz}, \mathrm{DMSO}-d_{6}, \mathrm{ppm}\right) \delta 2.62(2 \mathrm{H}, \mathrm{t}, J$ $=4.4 \mathrm{~Hz}$, piperazine protons), $2.64-2.67(2 \mathrm{H}, \mathrm{m}$, piperazine protons), 3.17 and $3.63\left(2 \mathrm{H}\right.$, two s, CO-CH $\left.\mathrm{CH}_{2}\right), 3.27(1 \mathrm{H}, \mathrm{s}$, piperazine proton), $3.42-3.52(3 \mathrm{H}, \mathrm{m}$, piperazine protons), $6.99\left(2 \mathrm{H}, \mathrm{dd}, J_{1}=9.6 \mathrm{~Hz}, J_{2}=4 \mathrm{~Hz}\right.$, phenyl protons $), 7.42-7.48$ (1H, m, phenyl proton), 7.62-7.66 (1H, m, phenyl proton), 7.92 $(1 \mathrm{H}, \mathrm{t}, J=8.4 \mathrm{~Hz}$, phenyl proton), 8.00-8.05 (2H, m, phenyl protons), 8.27 and $8.65(1 \mathrm{H}, 2 \mathrm{~s}, \mathrm{~N}=\mathrm{CH}), 11.50$ and $11.53(1 \mathrm{H}$, two s, NH).

${ }^{13}$ C-NMR (100 Mhz, DMSO- $d_{6}$, ppm) $\delta 46.18$ and 46.41 $\left(2 \mathrm{CH}_{2}\right), 51.97$ and $52.17\left(2 \mathrm{CH}_{2}\right), 56.79$ and $60.12\left(\mathrm{COCH}_{2}\right)$, 112.50 and $112.57(2 \mathrm{CH}), 125.60(2 \mathrm{CH}), 127.86(\mathrm{C}), 128.00(\mathrm{C})$, $129.25(\mathrm{C}), 130.48$ and $130.67(\mathrm{C}), 133.44$ and $133.71(\mathrm{C}), 134.66$ and $134.93(\mathrm{C}), 136.77$ and $136.87(\mathrm{C}), 137.87$ and $141.90(\mathrm{CH})$, $154.61(\mathrm{C}), 165.79$ and $170.79(\mathrm{C})$.

HRMS $(m / z):[\mathrm{M}+\mathrm{H}]^{+}$calcd for $\mathrm{C}_{19} \mathrm{H}_{19} \mathrm{Cl}_{2} \mathrm{~N}_{5} \mathrm{O}_{3}$ : 436.0938; found 436.0928.

2.4.6. $N^{\prime}$-(4-Cyanobenzylidene)-2-(4-(4-nitrophenyl)piperazin-1-yl)acetohydrazide (3f). IR $(\mathrm{KBr}) v_{\max }\left(\mathrm{cm}^{-1}\right): 3261(\mathrm{~N}-$ $\mathrm{H}), 3065$ (Aromatic C-H), 2833 (Aliphatic C-H), $2223(\mathrm{C} \equiv \mathrm{N})$, $1683(\mathrm{C}=\mathrm{O}), 1597$ and $1305\left(\mathrm{NO}_{2}\right), 1508(\mathrm{C}=\mathrm{N}), 1471(\mathrm{C}=\mathrm{C})$, 1240 (C-N), 1087 and 825 (C-H out of plane deformation).
${ }^{1} \mathrm{H}-\mathrm{NMR}$ (400 Mhz, DMSO- $\left.d_{6}, \mathrm{ppm}\right) \delta 2.66(2 \mathrm{H}, \mathrm{t}, J=$ $4.8 \mathrm{~Hz}$, piperazine protons), $2.74(2 \mathrm{H}, \mathrm{t}, J=4.8 \mathrm{~Hz}$, piperazine protons), 3.22 and $3.68\left(2 \mathrm{H}\right.$, two s, CO- $\left.\mathrm{CH}_{2}\right), 3.37(1 \mathrm{H}, \mathrm{s}$, piperazine proton), 3.49-3.58 (3H, $\mathrm{m}$, piperazine protons), $7.03\left(2 \mathrm{H}, \mathrm{dd}, J_{1}=9.2 \mathrm{~Hz}, J_{2}=4 \mathrm{~Hz}\right.$, phenyl protons), 7.82-7.92 (4H, m, phenyl protons), 8.01 and $8.40(1 \mathrm{H}$, two s, $\mathrm{N}=\mathrm{CH})$, $8.05\left(2 \mathrm{H}, \mathrm{dd}, J_{1}=9.2 \mathrm{~Hz}, J_{2}=3.2 \mathrm{~Hz}\right.$, phenyl protons), 11.51 and $11.59(1 \mathrm{H}$, two s, NH).

${ }^{13}$ C-NMR (100 Mhz, DMSO- $d_{6}$, ppm) $\delta 46.15$ and 46.35 $\left(2 \mathrm{CH}_{2}\right), 51.98$ and $52.15\left(2 \mathrm{CH}_{2}\right), 56.80$ and $60.02\left(\mathrm{COCH}_{2}\right)$, 111.50 and $111.78(\mathrm{CN}), 112.47$ and $112.54(2 \mathrm{CH}), 118.5 \overline{6}$ and $118.61(\mathrm{C}), 125.62(2 \mathrm{CH}), 127.21$ and $127.48(2 \mathrm{CH}), 132.62$ (2CH), 136.71 and $136.82(\mathrm{C}), 138.54$ and $138.70(\mathrm{C}), 140.89$ and $145.14(\mathrm{C}=\mathrm{N}), 154.58$ and $154.64(\mathrm{C}), 165.84$ and 170.95 $(\mathrm{C}=\mathrm{O})$. HRMS $(m / z):[\mathrm{M}+\mathrm{H}]^{+}$calcd for $\mathrm{C}_{20} \mathrm{H}_{20} \mathrm{~N}_{6} \mathrm{O}_{3}$ : 393.1670; found 393.1654.

2.4.7. 2-(4-(4-Nitrophenyl)piperazin-1-yl)- $N^{\prime}$-(4-(trifluoromethyl)benzylidene)acetohydrazide (3g). IR (KBr) $v_{\max }$ $\left(\mathrm{cm}^{-1}\right)$ : $3267(\mathrm{~N}-\mathrm{H}), 3035$ (Aromatic C-H), 2835 (Aliphatic $\mathrm{C}-\mathrm{H}), 1683(\mathrm{C}=\mathrm{O}), 1597$ and $1303\left(\mathrm{NO}_{2}\right), 1506(\mathrm{C}=\mathrm{N}), 1473$ $(\mathrm{C}=\mathrm{C}), 1242(\mathrm{C}-\mathrm{N}), 1116,1083$ and $825(\mathrm{C}-\mathrm{H}$ out of plane deformation).

${ }^{1} \mathrm{H}-\mathrm{NMR}$ (400 Mhz, DMSO- $\left.d_{6}, \mathrm{ppm}\right) \delta 2.67(2 \mathrm{H}, \mathrm{t}, J=$ $4.8 \mathrm{~Hz}$, piperazine protons), $2.75(2 \mathrm{H}, \mathrm{t}, J=4.4 \mathrm{~Hz}$, piperazine protons), 3.22 and $3.69\left(2 \mathrm{H}\right.$, two s, CO- $\left.\mathrm{CH}_{2}\right), 3.38(1 \mathrm{H}, \mathrm{s}$, piperazine proton), 3.46-3.56 (3H, $\mathrm{m}$, piperazine protons), 7.01-7.06 (2H, m, phenyl protons), $7.79(2 \mathrm{H}, \mathrm{t}, J=8.4 \mathrm{~Hz}$, phenyl protons), 7.86-8.09 (2H, m, phenyl protons), $8.06(2 \mathrm{H}$, $\mathrm{dd}, J_{1}=9.2 \mathrm{~Hz}, J_{2}=3.6 \mathrm{~Hz}$, phenyl protons $), 8.43(1 \mathrm{H}, \mathrm{s}$, $\mathrm{C}=\mathrm{N}), 11.47$ and $11.56(1 \mathrm{H}$, two $\mathrm{s}, \mathrm{NH})$.

${ }^{13} \mathrm{C}-\mathrm{NMR}$ (100 Mhz, DMSO- $d_{6}$, ppm) $\delta 46.19$ and 46.40 $\left(2 \mathrm{CH}_{2}\right), 52.02$ and $52.21\left(2 \mathrm{CH}_{2}\right), 56.86$ and $60.08\left(\mathrm{COCH}_{2}\right)$, 112.51 and $112.59(2 \mathrm{CH}), 122.69\left(\mathrm{CF}_{3}\right), 125.40$ and $\overline{125.60}$ $(2 \mathrm{CH}), 125.65(2 \mathrm{CH}), 127.24$ and $127.55(2 \mathrm{CH}), 129.22,129.45$, 129.53, 129.77 (C), 136.76 and 136.87 (C), 138.09 and 138.25 (C), 141.20 and $145.41(\mathrm{C}=\mathrm{N}), 154.64$ and $154.69(\mathrm{C}), 165.81$ and $170.91(\mathrm{C}=\mathrm{O})$.

HRMS $(m / z):[\mathrm{M}+\mathrm{H}]^{+}$calcd for $\mathrm{C}_{20} \mathrm{H}_{20} \mathrm{~F}_{3} \mathrm{~N}_{5} \mathrm{O}_{3}$ : 436.1591; found 436.1577 .

2.4.8. $\quad N^{\prime}$-(4-(Dimethylamino)benzylidene)-2-(4-(4-nitrophenyl)piperazin-1-yl)acetohydrazide (3h). IR (KBr) $v_{\max }$ $\left(\mathrm{cm}^{-1}\right)$ : $3250(\mathrm{~N}-\mathrm{H}), 3078$ (Aromatic C-H), 2962 (Aliphatic $\mathrm{C}-\mathrm{H}), 1681(\mathrm{C}=\mathrm{O}), 1583$ and $1315\left(\mathrm{NO}_{2}\right), 1595(\mathrm{C}=\mathrm{N}), 1477$ $(\mathrm{C}=\mathrm{C}), 1232(\mathrm{C}-\mathrm{N}), 1114,1093$ and $827(\mathrm{C}-\mathrm{H}$ out of plane deformation).

${ }^{1} \mathrm{H}-\mathrm{NMR}\left(400 \mathrm{Mhz}, \mathrm{DMSO}-d_{6}, \mathrm{ppm}\right) \delta 1.21\left(6 \mathrm{H}, \mathrm{dd}, J_{1}\right.$ $\left.=7.2 \mathrm{~Hz}, J_{2}=2.8 \mathrm{~Hz}, \mathrm{~N}\left(\mathrm{CH}_{3}\right)_{2}\right), 2.65(2 \mathrm{H}, \mathrm{t}, J=4.8 \mathrm{~Hz}$, piperazine protons), $2.74(2 \mathrm{H}, \mathrm{t}, J=4.8 \mathrm{~Hz}$, piperazine protons), 3.18 and $3.65\left(2 \mathrm{H}\right.$, two s, CO-CH $\left.{ }_{2}\right), 3.39(1 \mathrm{H}, \mathrm{s}$, piperazine proton), 3.44-3.56 (3H, $\mathrm{m}$, piperazine protons), $7.00-7.05(2 \mathrm{H}, \mathrm{m}$, phenyl protons), $7.30(2 \mathrm{H}, \mathrm{t}, J=8.2 \mathrm{~Hz}$, phenyl protons), $7.58(1 \mathrm{H}, \mathrm{d}, J=8 \mathrm{~Hz}$, phenyl protons), 7.62 ( $1 \mathrm{H}, \mathrm{d}, J=8 \mathrm{~Hz}$, phenyl proton), 7.96 and 8.32 (1H, two s, $\mathrm{N}=\mathrm{CH}), 8.05\left(2 \mathrm{H}, \mathrm{dd}, J_{1}=9.2 \mathrm{~Hz}, J_{2}=3.6 \mathrm{~Hz}\right.$, phenyl protons), 11.21 and 11.31 (1H, two s, NH). 
${ }^{13}$ C-NMR (100 Mhz, DMSO- $d_{6}$, ppm) $\delta 23.56$ and 33.29 $\left(2 \mathrm{CH}_{3}\right), 46.18$ and $46.38\left(2 \mathrm{CH}_{2}\right), 52.03$ and $52.20\left(2 \mathrm{CH}_{2}\right)$, 56.87 and $60.09\left(\mathrm{COCH}_{2}\right), 112.47$ and $112.55(2 \mathrm{CH}), 125.63$ $(2 \mathrm{CH}), 126.69$ and $126.73(2 \mathrm{CH}), 127.07(\mathrm{C}), 131.81$ and 131.89 $(2 \mathrm{CH}), 136.71$ and $136.83(\mathrm{C}), 142.91$ and $147.20(\mathrm{C}=\mathrm{N}), 150.26$ and $150.54(\mathrm{C}), 154.61$ and $154.66(\mathrm{C}), 165.33$ and $170.51(\mathrm{C})$.

HRMS $(m / z):[\mathrm{M}+\mathrm{H}]^{+}$calcd for $\mathrm{C}_{22} \mathrm{H}_{28} \mathrm{~N}_{6} \mathrm{O}_{3}: 424.4961$; found 424.4967 .

2.4.9. $N^{\prime}$-(4-(Diethylamino)benzylidene)-2-(4-(4-nitrophenyl) piperazin-1-yl)acetohydrazide (3i). IR (KBr) $v_{\max }\left(\mathrm{cm}^{-1}\right)$ : $3203(\mathrm{~N}-\mathrm{H}), 3049$ (Aromatic C-H), 2985 (Aliphatic C-H), $1660(\mathrm{C}=\mathrm{O}), 1587$ and $1315\left(\mathrm{NO}_{2}\right), 1548(\mathrm{C}=\mathrm{N}), 1494(\mathrm{C}=\mathrm{C})$, 1240 (C-N), 1195, 1097, 815 (C-H out of plane deformation).

${ }^{1} \mathrm{H}-\mathrm{NMR}$ (400 Mhz, DMSO- $d_{6}$, ppm) $\delta 2.60(2 \mathrm{H}, \mathrm{t}, J=$ $4.8 \mathrm{~Hz}$, piperazine protons), $2.70(2 \mathrm{H}, \mathrm{t}, J=4.8 \mathrm{~Hz}$, piperazine protons), 3.09 and $3.56\left(2 \mathrm{H}\right.$, two s, $\left.\mathrm{CO}-\mathrm{CH}_{2}\right), 3.28-3.38(10 \mathrm{H}$, $\left.\mathrm{m}, \mathrm{N}\left(\mathrm{C}_{2} \mathrm{H}_{5}\right)_{2}\right), 3.43-3.55$ (4H, m, piperazine protons), 6.65 $(2 \mathrm{H}, \mathrm{t}, J=9.2 \mathrm{~Hz}$, phenyl protons $), 7.01\left(2 \mathrm{H}, \mathrm{dd}, J_{1}=9.2 \mathrm{~Hz}, J_{2}\right.$ $=3.2 \mathrm{~Hz}$, phenyl protons), 7.38-7.45 ( $2 \mathrm{H}, \mathrm{m}$, phenyl protons), 7.78 and $8.10(1 \mathrm{H}$, two s, $\mathrm{N}=\mathrm{CH}), 8.03\left(2 \mathrm{H}, \mathrm{dd}, J_{1}=9.6 \mathrm{~Hz}, J_{2}\right.$ $=3.2 \mathrm{~Hz}$, phenyl protons), 10.86 and $10.98(1 \mathrm{H}$, two s, NH).

${ }^{13} \mathrm{C}-\mathrm{NMR}\left(100 \mathrm{Mhz}, \mathrm{DMSO}-d_{6}, \mathrm{ppm}\right) \delta 12.31\left(2 \mathrm{CH}_{3}\right)$, $43.58\left(2 \mathrm{CH}_{2}\right), 46.20$ and $46.39\left(2 \mathrm{CH}_{2}\right), 52.00$ and 52.14 $\left(2 \mathrm{CH}_{2}\right), 56.83$ and $60.08\left(\mathrm{COCH}_{2}\right), 111.00$ and $111.06(2 \mathrm{CH})$, 112.45 and $112.53(2 \mathrm{CH}), 120.54$ and $120.61(\mathrm{C}), 125.57(2 \mathrm{CH})$, 128.17 and $128.57(2 \mathrm{CH}), 136.71$ and $136.83(\mathrm{C}), 143.69$ and $148.04(\mathrm{C}=\mathrm{N}), 148.54$ and $148.76(\mathrm{C}), 154.60$ and $154.65(\mathrm{C})$, 164.58 and $169.91(\mathrm{C}=\mathrm{O})$.

HRMS $(m / z):[\mathrm{M}+\mathrm{H}]^{+}$calcd for $\mathrm{C}_{23} \mathrm{H}_{30} \mathrm{~N}_{6} \mathrm{O}_{3}$ : 439.2452; found 439.2436 .

2.4.10. $N^{\prime}$-(4-Methylbenzylidene)-2-(4-(4-nitrophenyl)piperazin-1-yl)acetohydrazide (3j). IR (KBr) $v_{\max }\left(\mathrm{cm}^{-1}\right): 3255(\mathrm{~N}-$ $\mathrm{H}), 3082$ (Aromatic C-H), 2829 (Aliphatic C-H), $1683(\mathrm{C}=\mathrm{O})$, 1589 and $1319\left(\mathrm{NO}_{2}\right), 1508(\mathrm{C}=\mathrm{N}), 1489(\mathrm{C}=\mathrm{C}), 1240(\mathrm{C}-\mathrm{N})$, 1089, 823 (C-H out of plane deformation).

${ }^{1} \mathrm{H}-\mathrm{NMR}$ (400 Mhz, DMSO- $\left.d_{6}, \mathrm{ppm}\right) \delta 2.31\left(3 \mathrm{H}, \mathrm{s}, \mathrm{CH}_{3}\right)$, $2.62(2 \mathrm{H}, \mathrm{t}, J=4.8 \mathrm{~Hz}$, piperazine protons $), 2.71(2 \mathrm{H}, \mathrm{t}, J=$ $4.8 \mathrm{~Hz}$, piperazine protons), 3.14 and $3.61(2 \mathrm{H}$, two s, CO$\left.\mathrm{CH}_{2}\right), 3.45(2 \mathrm{H}, \mathrm{t}, J=4.8 \mathrm{~Hz}$, piperazine protons, $3.49(2 \mathrm{H}$, $\mathrm{t}, J=4.8 \mathrm{~Hz}$, piperazine protons $), 6.99\left(2 \mathrm{H}, \mathrm{dd}, J_{1}=9.6 \mathrm{~Hz}\right.$, $J_{2}=4 \mathrm{~Hz}$, phenyl protons), $7.21(2 \mathrm{H}, \mathrm{t}, J=7.2 \mathrm{~Hz}$, phenyl protons), $7.50-7.57(2 \mathrm{H}, \mathrm{m}$, phenyl protons), 7.91 and $8.27(1 \mathrm{H}$, two s, $\mathrm{N}=\mathrm{CH}), 8.02\left(2 \mathrm{H}\right.$, dd, $J_{1}=9.2 \mathrm{~Hz}, J_{2}=3.6 \mathrm{~Hz}$, phenyl protons), 11.11 and $11.19(1 \mathrm{H}$, two s, $\mathrm{NH})$.

${ }^{13} \mathrm{C}-\mathrm{NMR}$ (100 Mhz, DMSO-d $\left.d_{6}, \mathrm{ppm}\right) \delta 20.87\left(\mathrm{CH}_{3}\right)$, 46.18 and $46.38\left(2 \mathrm{CH}_{2}\right), 51.96$ and $52.13\left(2 \mathrm{CH}_{2}\right), 56.79$ and $60.04\left(\mathrm{COCH}_{2}\right), 112.45$ and $112.53(2 \mathrm{CH}), 125.56(2 \mathrm{CH})$, 126.57 and $126.88(2 \mathrm{CH}), 129.27(2 \mathrm{CH}), 131.38$ and $131.48(\mathrm{C})$, 136.73 and $136.85(\mathrm{C}), 139.37$ and $139.68(\mathrm{C}), 142.90$ and 147.19 $(\mathrm{C}=\mathrm{N}), 154.58$ and $154.63(\mathrm{C}), 165.23$ and $170.44(\mathrm{C}=\mathrm{O})$.

HRMS $(m / z):[\mathrm{M}+\mathrm{H}]^{+}$calcd for $\mathrm{C}_{20} \mathrm{H}_{23} \mathrm{~N}_{5} \mathrm{O}_{3}$ : 382.1874; found 382.1885 .

2.4.11. $N^{\prime}$-(4-(Methylthio)benzylidene)-2-(4-(4-nitrophenyl)piperazin-1-yl)acetohydrazide (3k). IR $(\mathrm{KBr}) \nu_{\max }\left(\mathrm{cm}^{-1}\right): 3244$ (N-H), 3055 (Aromatic C-H), 2833 (Aliphatic C-H), 1683
$(\mathrm{C}=\mathrm{O}), 1593$ and $1307\left(\mathrm{NO}_{2}\right), 1508(\mathrm{C}=\mathrm{N}), 1471(\mathrm{C}=\mathrm{C}), 1242$ (C-N), 1116, 1087, 819 (C-H out of plane deformation).

${ }^{1} \mathrm{H}-\mathrm{NMR}$ (400 Mhz, DMSO- $\left.d_{6}, \mathrm{ppm}\right) \delta 2.51(3 \mathrm{H}, \mathrm{s}$, $\left.\mathrm{SCH}_{3}\right), 2.63-2.68(2 \mathrm{H}, \mathrm{m}$, piperazine protons $), 2.72-2.76(2 \mathrm{H}$, $\mathrm{m}$, piperazine protons), 3.18 and $3.65\left(2 \mathrm{H}, 2 \mathrm{~s}, \mathrm{CO}-\mathrm{CH}_{2}\right), 3.33$ (1H, br s, piperazine proton), 3.44-3.56 (3H, m, piperazine protons), $7.01\left(2 \mathrm{H}, \mathrm{dd}, J_{1}=9.6 \mathrm{~Hz}, J_{2}=4 \mathrm{~Hz}\right.$, phenyl protons), $7.30(2 \mathrm{H}, \mathrm{t}, J=7.6 \mathrm{~Hz}$, phenyl protons), 7.57-7.64 $(2 \mathrm{H}, \mathrm{m}$, phenyl protons), 7.94 and $8.30(1 \mathrm{H}$, two s, $\mathrm{N}=\mathrm{CH}), 8.05(2 \mathrm{H}$, dd, $J_{1}=9.6 \mathrm{~Hz}, J_{2}=3.6 \mathrm{~Hz}$, phenyl protons), 11.17 and 11.26 $(1 \mathrm{H}$, two s, NH).

${ }^{13} \mathrm{C}-\mathrm{NMR}\left(100 \mathrm{Mhz}, \mathrm{DMSO}-d_{6}, \mathrm{ppm}\right) \delta 14.40\left(\mathrm{CH}_{3}\right)$, 46.18 and $46.37\left(2 \mathrm{CH}_{2}\right), 51.97$ and $52.13\left(2 \mathrm{CH}_{2}\right), 56.80$ and $60.04\left(\mathrm{COCH}_{2}\right), 112.42$ and $112.50(2 \mathrm{CH}), 125.55$ and 125.58 (C), $125.63(2 \mathrm{CH}), 127.00$ and $127.31(2 \mathrm{CH}), 130.60$ and 130.65 $(2 \mathrm{CH}), 136.74$ and $136.85(\mathrm{C}), 140.40$ and $140.77(\mathrm{C}), 142.43$ and $146.76(\mathrm{C}=\mathrm{N}), 154.56$ and $154.60(\mathrm{C}), 165.24$ and 170.44 $(\mathrm{C}=\mathrm{O})$. HRMS $(\mathrm{m} / z):[\mathrm{M}+\mathrm{H}]^{+}$calcd for $\mathrm{C}_{20} \mathrm{H}_{23} \mathrm{~N}_{5} \mathrm{O}_{3} \mathrm{~S}$ : 414.1594; found 414.1589 .

2.5. Enzymatic Assay. AChE and BuChE inhibitory activity was determined by Ellman's method with minor modifications [22]. Compounds 3a-k were dissolved in 2\% DMSO and were tested at final concentrations of 5, 10, 20, 40, and $80 \mathrm{mg} / \mathrm{mL}$. $20 \mathrm{~mL}$ (1 unit/mL) of AChE (from Electrophorus electricus, electric eel) or BuChE (from equine serum) and $10 \mathrm{~mL}$ sample were added to $2.4 \mathrm{~mL}$ buffer; the mixture was incubated at $37^{\circ} \mathrm{C}$ for $15 \mathrm{~min}$. After the $15 \mathrm{~min}$ incubation, $50 \mathrm{~mL}$ of $0.01 \mathrm{M}$ DTNB and $20 \mathrm{~mL}$ of $75 \mathrm{mM}$ ATCI or $10 \mathrm{mM}$ BTCI were added, and the final mixture was incubated at room temperature for $30 \mathrm{~min}$. A control mixture (blank) was prepared using $10 \mathrm{~mL}$ of $2 \%$ DMSO instead of the test sample, with all other procedures similar to those used in the case of the sample mixture. Absorbance was measured at $412 \mathrm{~nm}$ and $37^{\circ} \mathrm{C}$ using polystyrol cuvettes with spectrophotometer (Shimadzu, UV-1700). Experiment was done in triplicate. Data are expressed as mean standard deviation (SD). The inhibition (percent) of $\mathrm{AChE}$ and $\mathrm{BuChE}$ was calculated using the following equation. The statistical analysis was evaluated using Microsoft Office Excel 2013 and the data were expressed as mean $\pm \mathrm{SD}$ :

$$
I(\%)=100-\left(\frac{\mathrm{OD}_{\text {sample }}}{\mathrm{OD}_{\text {control }}}\right) \times 100
$$

\section{Results and Discussion}

3.1. Chemistry. The synthesis of the compounds (3a-k) was carried out in three steps as shown in Figure 1. In the first step, ethyl 2-[4-(4-nitrophenyl)piperazin-1-yl]acetate (1) was synthesized via the reaction of 1-(4-nitrophenyl)piperazine with ethyl chloroacetate in the existence of potassium carbonate. To obtain corresponding hydrazide (2) compound 1 reacted with excess of hydrazine hydrate in ethanol. Finally, the reaction of 2-[4-(4-nitrophenyl)piperazin-1yl]acetohydrazide (2) with aromatic aldehydes gave the target compounds $(\mathbf{3} \mathbf{a}-\mathbf{k})$. Some characteristic properties of the compounds were given in Table 1. 
TABLE 1: Some properties of the compounds (3a-k).

\begin{tabular}{|c|c|c|c|c|c|c|}
\hline Compound & $\mathrm{R}$ & $\mathrm{R}^{\prime}$ & Yield (\%) & $\mathrm{Mp}\left({ }^{\circ} \mathrm{C}\right)$ & Molecular formula & Molecular weight \\
\hline $3 a$ & $\mathrm{H}$ & $\mathrm{F}$ & 74 & 181 & $\mathrm{C}_{19} \mathrm{H}_{20} \mathrm{FN}_{5} \mathrm{O}_{3}$ & 385 \\
\hline $3 b$ & $\mathrm{H}$ & $\mathrm{OCH}_{3}$ & 80 & 207 & $\mathrm{C}_{20} \mathrm{H}_{23} \mathrm{~N}_{5} \mathrm{O}_{4}$ & 397 \\
\hline $3 c$ & $\mathrm{H}$ & $\mathrm{OH}$ & 70 & 262 & $\mathrm{C}_{19} \mathrm{H}_{21} \mathrm{~N}_{5} \mathrm{O}_{4}$ & 383 \\
\hline $3 d$ & $\mathrm{H}$ & $\mathrm{OC}_{2} \mathrm{H}_{5}$ & 76 & 204 & $\mathrm{C}_{21} \mathrm{H}_{25} \mathrm{~N}_{5} \mathrm{O}_{4}$ & 411 \\
\hline $3 e$ & $\mathrm{Cl}$ & $\mathrm{Cl}$ & 72 & 165 & $\mathrm{C}_{19} \mathrm{H}_{19} \mathrm{Cl}_{2} \mathrm{~N}_{5} \mathrm{O}_{3}$ & 436 \\
\hline $3 f$ & $\mathrm{H}$ & $\mathrm{CN}$ & 78 & 226 & $\mathrm{C}_{20} \mathrm{H}_{20} \mathrm{~N}_{6} \mathrm{O}_{3}$ & 392 \\
\hline $3 g$ & $\mathrm{H}$ & $\mathrm{CF}_{3}$ & 82 & 212 & $\mathrm{C}_{20} \mathrm{H}_{20} \mathrm{~F}_{3} \mathrm{~N}_{5} \mathrm{O}_{3}$ & 435 \\
\hline $3 h$ & $\mathrm{H}$ & $\mathrm{N}\left(\mathrm{CH}_{3}\right)_{2}$ & 75 & 95 & $\mathrm{C}_{21} \mathrm{H}_{26} \mathrm{~N}_{6} \mathrm{O}_{3}$ & 410 \\
\hline $3 \mathbf{i}$ & $\mathrm{H}$ & $\mathrm{N}\left(\mathrm{C}_{2} \mathrm{H}_{5}\right)_{2}$ & 78 & 221 & $\mathrm{C}_{23} \mathrm{H}_{30} \mathrm{~N}_{6} \mathrm{O}_{3}$ & 438.5 \\
\hline $3 \mathbf{j}$ & $\mathrm{H}$ & $\mathrm{CH}_{3}$ & 73 & 227 & $\mathrm{C}_{20} \mathrm{H}_{23} \mathrm{~N}_{5} \mathrm{O}_{3}$ & 381 \\
\hline $3 k$ & $\mathrm{H}$ & $\mathrm{SCH}_{3}$ & 81 & 203 & $\mathrm{C}_{20} \mathrm{H}_{23} \mathrm{~N}_{5} \mathrm{O}_{3} \mathrm{~S}$ & 413.5 \\
\hline
\end{tabular}<smiles>CCOC(=O)CCl</smiles><smiles>CCOC(=O)CN1CCN(c2ccc([N+](=O)[O-])cc2)CC1</smiles><smiles>[R]c1ccc(/C=N/NC(=O)CN2CCN(c3ccc([N+](=O)[O-])cc3)CC2)c([R])c1</smiles><smiles>NNC(=O)CN1CCN(c2ccc([N+](=O)[O-])cc2)CC1</smiles>

FIGURE 1: Synthesis of the compounds (3a-k). Reactants, reagents, and conditions; (a) $\mathrm{K}_{2} \mathrm{CO}_{3}$, acetone rt; (b) $\mathrm{NH}_{2} \mathrm{NH}_{2} \cdot \mathrm{H}_{2} \mathrm{O}$, ethanol, rt; (c) aromatic aldehyde, ethanol, reflux.

Structures of the obtained compounds were elucidated by ${ }^{1} \mathrm{H}$ NMR, ${ }^{13} \mathrm{C}$ NMR, and HRMS spectral data.

In the IR spectra of compounds (3a-k), significant stretching bands belonging to $\mathrm{C}=\mathrm{O}$ were observed in between $1651-1695 \mathrm{~cm}^{-1}$, bands belonging to $\mathrm{C}=\mathrm{N}$ and $\mathrm{C}=\mathrm{C}$ were observed in between $1599-1471 \mathrm{~cm}^{-1}$, and bands belonging to $\mathrm{NO}_{2}$ were observed at about 1597.06 and $1303.88 \mathrm{~cm}^{-1}$.

In the ${ }^{1} \mathrm{H}$ NMR spectra of the final compounds (3a$\mathbf{k})$, the hydrazone proton was observed in the area of 10.5$12 \mathrm{ppm}$. The protons belonging to $\mathrm{CO}-\mathrm{CH}_{2}(3.09-3.56 \mathrm{ppm})$, $\mathrm{N}=\mathrm{CH}(7.78-8.10 \mathrm{ppm})$, and $\mathrm{CO}-\mathrm{NH}(11.03-11.13 \mathrm{ppm})$ were detected as paired peaks with respect to the presence of the $E$ and $Z$ forms of the compounds $[18,19]$. The other aromatic and aliphatic protons were observed at the expected regions.
In ${ }^{13} \mathrm{C}$ NMR spectra of the compounds, the carbons belonging to $-\mathrm{N}=\mathrm{CH}-(140-147 \mathrm{ppm})$, carbonyl (165$170 \mathrm{ppm}$ ), and $-\mathrm{CH}_{2}$ - attached to carbonyl (55-60 ppm) were observed as paired peaks due to $E$ and $Z$ isomer forms. Due to the presence of fluoro in compound $\mathbf{3 a}$ and $\mathbf{3 g}$, splitting relating to neighbouring atoms was detected in the spectrum.

In the HRMS spectra of final compounds (3a-k), the M +1 peak was observed in accordance with their molecular formula. The $\mathrm{M}+1$ peaks were determined in accuracy of 5$16 \mathrm{ppm}$.

3.2. Enzyme Inhibition. Therapeutic efficacy of novel hydrazone derivatives for the treatment of $\mathrm{AD}$ depends on their anticholinesterase activities. Inhibition of $\mathrm{AChE}$ and BuChE 
TABLE 2: Cholinesterase inhibitory activity and $\mathrm{IC}_{50}$ values $(\mu \mathrm{g} / \mathrm{mL})$ of compounds $(\mathbf{3 a}-\mathbf{k})$.

\begin{tabular}{|c|c|c|c|c|}
\hline \multirow{2}{*}{ Compound } & \multicolumn{2}{|c|}{ AChE } & \multicolumn{2}{|c|}{ BuChE } \\
\hline & $80 \mathrm{mg} / \mathrm{mL}$ (\% inhibition) & $\mathrm{IC}_{50} \mathrm{mg} / \mathrm{mL}$ & $80 \mathrm{mg} / \mathrm{mL}$ (\% inhibition) & $\mathrm{IC}_{50} \mathrm{mg} / \mathrm{mL}$ \\
\hline $3 a$ & $42.30 \pm 4.70$ & $>80$ & $40.11 \pm 3.72$ & $>80$ \\
\hline $3 b$ & $16.30 \pm 2.43$ & $>80$ & $20.16 \pm 2.86$ & $>80$ \\
\hline $3 c$ & $57.30 \pm 0.66$ & $29.5 \pm 2.12$ & $46.56 \pm 3.20$ & $>80$ \\
\hline $3 d$ & - & - & $15.53 \pm 3.84$ & $>80$ \\
\hline $3 e$ & $33.37 \pm 4.45$ & $>80$ & $33.07 \pm 3.41$ & $>80$ \\
\hline $3 f$ & $37.22 \pm 3.46$ & $>80$ & $27.63 \pm 0.86$ & $>80$ \\
\hline $3 g$ & $24.74 \pm 1.81$ & $>80$ & $31.60 \pm 2.05$ & $>80$ \\
\hline $3 h$ & $12.11 \pm 2.80$ & $>80$ & - & - \\
\hline $3 \mathbf{i}$ & $35.14 \pm 4.43$ & $>80$ & - & - \\
\hline $3 \mathbf{j}$ & $11.39 \pm 0.16$ & $>80$ & - & - \\
\hline $3 \mathbf{k}$ & $23.85 \pm 1.85$ & $>80$ & - & - \\
\hline Galantamine & nt & $0.46 \pm 0.08$ & nt & $6.28 \pm 0.32$ \\
\hline
\end{tabular}

-: not active.

nt: nontested.

by synthesized derivatives (3a-k) is compared with galantamine as reference drug and $\mathrm{IC}_{50}$ values of individual compounds are listed in Table 2. The anticholinesterase activities of the compounds were detected via a modification of Ellman's spectrophotometric method. All compounds showed less anticholinesterase potency than reference drugs. Only compound $3 \mathrm{c}$ with substituent hydroxyl at para position exhibited available inhibitory effect against AChE having $\mathrm{IC}_{50}$ value of $29.5 \pm 2.12$. In addition, compound $3 \mathrm{a}$, with substituent fluoro at para position also showed an inhibitory effect against $\mathrm{AChE}$ and $\mathrm{BuChE}$. This conclusion supports that the hydroxyl and fluoro substituents on phenyl ring at para positions may have a considerable influence on anticholinesterase activity. It could be considered that hydrogen bonds contribute to the activity of compounds $\mathbf{3 a}$ and 3c. Substitution at orto and para positions with chloro did not increase the activity. Compound $\mathbf{3 d}$ was found to be nonactive against either AChE or BChE. When compared with reference drugs and other derivatives, compound $\mathbf{3 j}$ exhibited the lowest anticholinesterase activity.

\section{Conclusion}

In the present paper, we synthesized some hydrazone derivatives and evaluated their AChE and BuChE enzyme inhibitory activities. The consequences verify that compound $3 c$, with an $\mathrm{IC}_{50}$ value of $29.5 \pm 2.12$, is the most promising inhibitory agent of $\mathrm{AChE}$ and BuChE among these compounds. As a result, it may be pointed out that hydroxyl and fluoro substituents at para position on the phenyl ring have a vital influence on anticholinesterase activity. Further investigations can be executed on the evaluation of new potent AChE and BuChE inhibitory agents bearing hydrazone moiety by modification of compound $3 c$.

\section{Competing Interests}

The authors declare that there are no competing interests regarding the publication of this paper.

\section{Acknowledgments}

This study was financially supported by Anadolu University Scientific Research Projects Fund, Project no. 1605S316.

\section{References}

[1] R. J. Castellani, R. K. Rolston, and M. A. Smith, "Alzheimer disease," Disease-a-Month, vol. 56, no. 9, pp. 484-546, 2010.

[2] A. V. Terry Jr. and J. J. Buccafusco, "The cholinergic hypothesis of age and Alzheimer's disease-related cognitive deficits: recent challenges and their implications for novel drug development," Journal of Pharmacology and Experimental Therapeutics, vol. 306, no. 3, pp. 821-827, 2003.

[3] D. P. Perl, "Neuropathology of Alzheimer's disease," Mount Sinai Journal of Medicine, vol. 77, no. 1, pp. 32-42, 2010.

[4] L. Yurttaş, Z. A. Kaplancıklı, and Y. Özkay, "Design, synthesis and evaluation of new thiazole-piperazines as acetylcholinesterase inhibitors," Journal of Enzyme Inhibition and Medicinal Chemistry, vol. 28, no. 5, pp. 1040-1047, 2013.

[5] M. D. Altintop, Z. A. Kaplancikli, A. Ozdemir, G. TuranZitouni, H. E. Temel, and G. Akalín, "Synthesis and anticholinesterase activity and cytotoxicity of novel amide derivatives," Chemistry in Life Sciences, vol. 345, no. 2, pp. 112-116, 2012.

[6] G. Turan-Zitouni, A. Ozdemir, Z. A. Kaplancikli, M. D. Altintop, H. E. Temel, and G. A. Çiftçi, "Synthesis and biological evaluation of some thiazole derivatives as new cholinesterase inhibitors," Journal of Enzyme Inhibition and Medicinal Chemistry, vol. 28, no. 3, pp. 509-514, 2013.

[7] F. Massoud and G. C. Léger, "Pharmacological treatment of Alzheimer disease," Canadian Journal of Psychiatry, vol. 56, no. 10, pp. 579-588, 2011. 
[8] B. T. Winslow, M. K. Onysko, C. M. Stob, and K. A. Hazlewood, "Treatment of Alzheimer disease," American Family Physician, vol. 83, no. 12, pp. 1403-1412, 2011.

[9] M. B. Čolović, D. Z. Krstić, T. D. Lazarević-Pašti, A. M. Bondžić, and V. M. Vasić, "Acetylcholinesterase inhibitors: pharmacology and toxicology," Current Neuropharmacology, vol. 11, no. 3, pp. 315-335, 2013.

[10] E. K. Perry, B. E. Tomlinson, G. Blessed, K. Bergmann, P. H. Gibson, and R. H. Perry, "Correlation of cholinergic abnormalities with senile plaques and mental test scores in senile dementia," British Medical Journal, vol. 2, no. 6150, pp. 1457-1459, 1978.

[11] S. Utku, M. Gökçe, I. Orhan, and M. F. Şahin, "Synthesis of novel 6-substituted-3(2H)-pyridazinone-2-acetyl-2-(substituted/-nonsubstituted benzal)hydrazone derivatives and acetylcholinesterase and butyrylcholinesterase inhibitory activities in vitro," Arzneimittel-Forschung, vol. 61, no. 1, pp. 1-7, 2011.

[12] V. Alptüzün, M. Prinz, V. Hörr et al., "Interaction of (benzylidene-hydrazono)-1,4-dihydropyridines with $\beta$-amyloid, acetylcholine, and butyrylcholine esterases," Bioorganic \& Medicinal Chemistry, vol. 18, no. 5, pp. 2049-2059, 2010.

[13] N. S. Gwaram, H. M. Ali, M. A. Abdulla et al., "Synthesis, characterization, X-ray crystallography, acetyl cholinesterase inhibition and antioxidant activities of some novel ketone derivatives of gallic hydrazide-derived Schiff bases," Molecules, vol. 17, no. 3, pp. 2408-2427, 2012.

[14] A. B. Özçelik, M. Gökçe, I. Orhan, F. Kaynak, and M. F. Şahin, "Synthesis and antimicrobial, acetylcholinesterase and butyrylcholinesterase inhibitory activities of novel ester and hydrazide derivatives of $3(2 \mathrm{H})$-pyridazinone," Arzneimittel-Forschung, vol. 60 , no. 7, pp. 452-458, 2010.

[15] T. Bunyapaiboonsri, O. Ramström, S. Lohmann, J.-M. Lehn, L. Peng, and M. Goeldner, "Dynamic deconvolution of a preequilibrated dynamic combinatorial library of acetylcholinesterase inhibitors," ChemBioChem, vol. 2, no. 6, pp. 438-444, 2001.

[16] K. Gholivand, Z. Hosseini, S. Farshadian, and H. NaderiManesh, "Synthesis, characterization, oxidative degradation, antibacterial activity and acetylcholinesterase/butyrylcholinesterase inhibitory effects of some new phosphorus(V) hydrazides," European Journal of Medicinal Chemistry, vol. 45, no. 11, pp. 5130-5139, 2010.

[17] P. W. Elsinghorst, C. M. González Tanarro, and M. Gütschow, "Novel heterobivalent tacrine derivatives as cholinesterase inhibitors with notable selectivity toward butyrylcholinesterase," Journal of Medicinal Chemistry, vol. 49, no. 25, pp. 75407544, 2006.

[18] P. Szymański, E. Zurek, and E. Mikiciuk-Olasik, "New tacrinehydrazinonicotinamide hybrids as acetylcholinesterase inhibitors of potential interest for the early diagnostics of Alzheimer's disease," Pharmazie, vol. 61, no. 4, pp. 269-273, 2006.

[19] P. Meena, V. Nemaysh, M. Khatri, A. Manral, P. M. Luthra, and M. Tiwari, "Synthesis, biological evaluation and molecular docking study of novel piperidine and piperazine derivatives as multi-targeted agents to treat Alzheimer's disease," Bioorganic \& Medicinal Chemistry, vol. 23, no. 5, pp. 1135-1148, 2015.

[20] L. Yurttaş, Y. Özkay, H. Karaca Gençer, and U. Acar, "Synthesis of some new thiazole derivatives and their biological activity evaluation," Journal of Chemistry, vol. 2015, Article ID 464379, 7 pages, 2015.

[21] U. A. Mohsen, Z. A. Kaplancikli, Y. Özkay, and L. Yurttä, "Synthesis and evaluation of anti-acetylcholinesterase activity of some benzothiazole based new piperazine-dithiocarbamate derivatives," Drug Research, vol. 65, no. 4, pp. 176-183, 2015.

[22] G. L. Ellman, K. D. Courtney, V. Andres Jr., and R. M. Featherstone, "A new and rapid colorimetric determination of acetylcholinesterase activity," Biochemical Pharmacology, vol. 7, no. 2, pp. 88-95, 1961. 

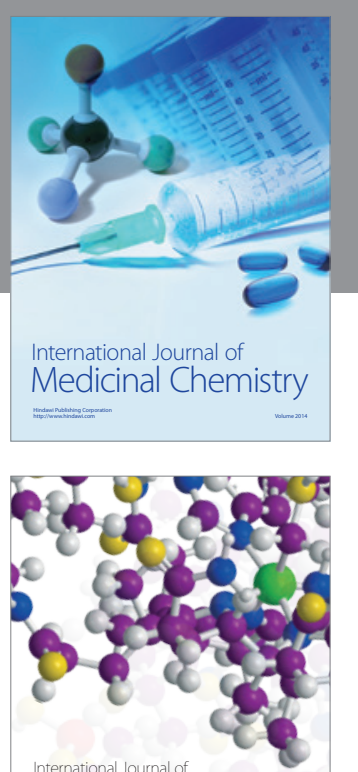

Carbohydrate Chemistry

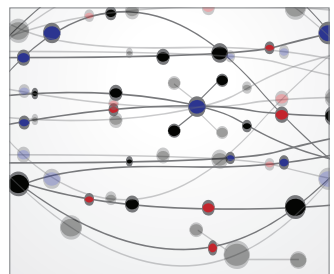

The Scientific World Journal
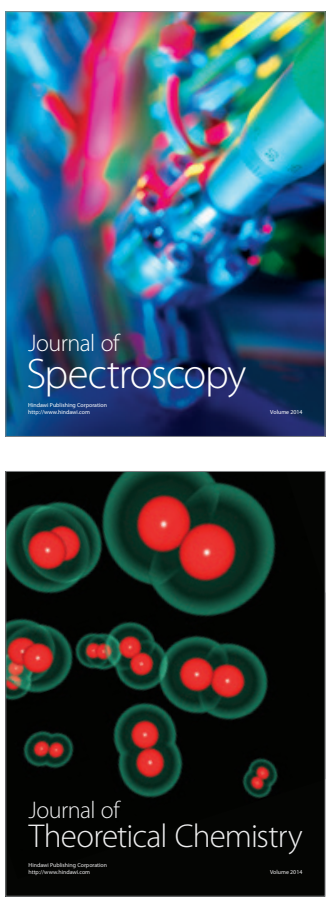
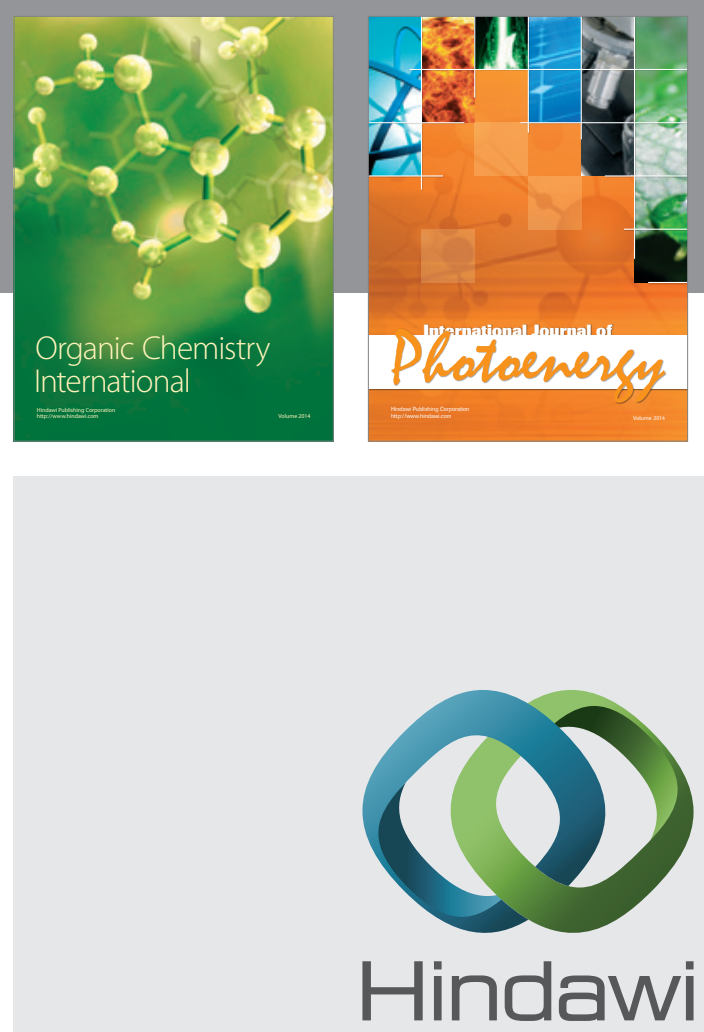

Submit your manuscripts at

http://www.hindawi.com

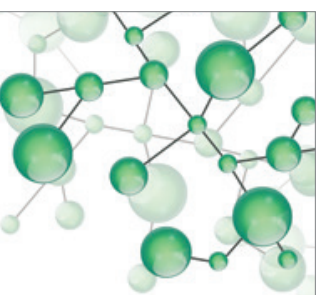

International Journal of

Inorganic Chemistry

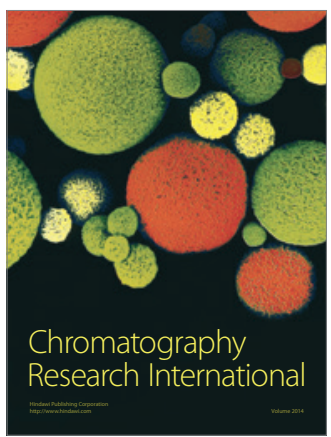

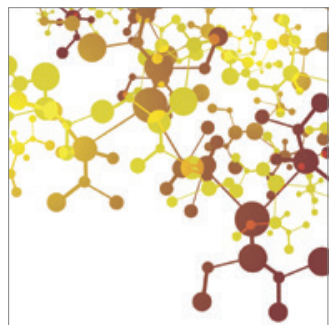

Applied Chemistry
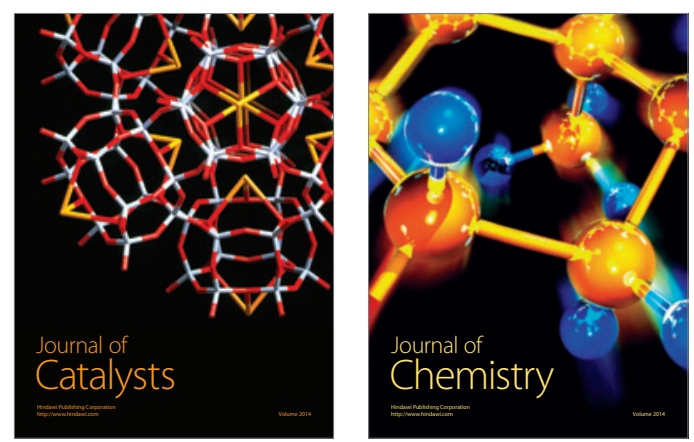
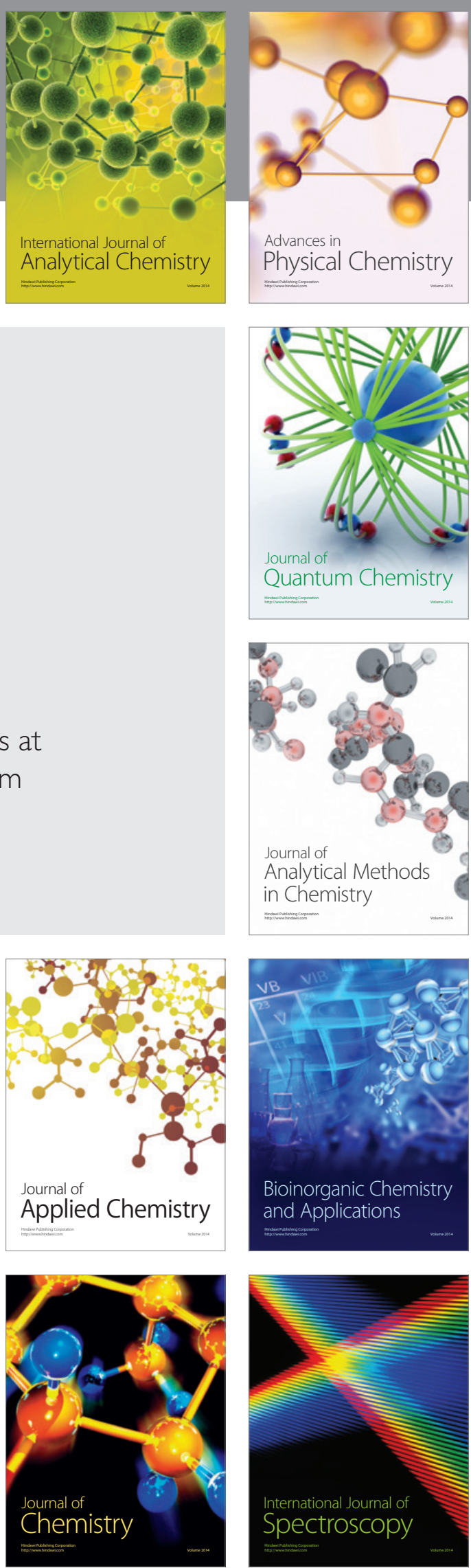